\title{
A Direct Catalytic Asymmetric Mannich-Type Reaction to Syn-Amino Alcohols.
}

\author{
Barry M. Trost* and Lamont R. Terrell \\ Department of Chemistry, Stanford University, Stanford, California 94305-5080.
}

\section{Experimental Procedure}

\section{General procedure for the imine aldol (Mannich-type) reaction of glyoxalates: Preparation of the catalyst:}

To a room temperature solution of ligand $1 \mathrm{a}(5 \mathrm{mg}, 0.0075 \mathrm{mmol})$ in THF $(0.3 \mathrm{~mL})$ was added dropwise a solution of diethylzinc ( $15 \square \mathrm{L}, 0.015 \mathrm{mmol}, 1.0 \mathrm{M}$ hexanes) and continued to stir for 30 minutes.

\section{Mannich-type (Imine Aldol) reaction:}

To a $-78{ }^{\circ} \mathrm{C}$ suspension of imine $\mathbf{4 b}(66 \mathrm{mg}, 0.30 \mathrm{mmol}$ ), 2-hydroxyacetophenone ( $82 \mathrm{mg}, 0.60$ $\mathrm{mmol})$ and crushed $4 \AA$ molecular sieves $(60 \mathrm{mg})$ in THF $(0.6 \mathrm{~mL})$ was added the zinc catalyst from above. After stirring at $-5^{\circ} \mathrm{C}$ for $6-12$ hours, the reaction mixture was diluted with $\mathrm{Et}_{2} \mathrm{O}(6 \mathrm{~mL})$ and then a phosphate buffer $(\mathrm{pH}=7)$ solution $(3 \mathrm{~mL})$. After stirring for 30 minutes, the phases were separated and the aqueous phase was extracted with $\mathrm{Et}_{2} \mathrm{O}(1 \mathrm{~mL} \times 2)$. The combined organics were washed with brine $(1.5 \mathrm{~mL})$, dried over $\mathrm{MgSO}_{4}$, filtered and concentrated to give an oil. The crude residue was purified by flash chromatography on silica gel [PE / EtOAc $10 \%$ 15\%] to give $87 \mathrm{mg} \mathrm{(81 \% )} \mathrm{of} \mathrm{imine} \mathrm{aldol} 5$ as a yellow oil.

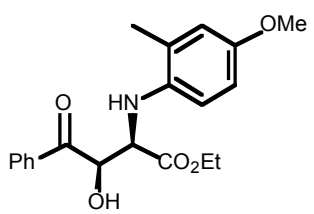

Syn diastereomer: $\mathrm{t}_{\mathrm{r}}=13.9 \mathrm{~min}$ (major enantiomer) and $21.1 \mathrm{~min}$, (Chiralcel OD, $\square=254 \mathrm{~nm}$, heptane: $\mathrm{i}-$ $\mathrm{PrOH}=90: 10,0.9 \mathrm{~mL} / \mathrm{min}$ ); $[\square]^{25}{ }_{\mathrm{D}}+46.3^{\circ}$ (c 0.659, $\mathrm{CHCl}_{3}, 93.6 \%$ ee); ${ }^{1} \mathrm{H} \mathrm{NMR}\left(300 \mathrm{MHz}, \mathrm{CDCl}_{3}\right) \square=7.92$ (d, $J=8.8 \mathrm{~Hz}, 2 \mathrm{H}), 7.64$ (t, $J=7.8 \mathrm{~Hz}, 1 \mathrm{H}), 7.52(\mathrm{t}, J=7.8 \mathrm{~Hz}, 2 \mathrm{H}), 6.71$ (d, $J=2.0 \mathrm{~Hz}, 1 \mathrm{H}), 6.64(\mathrm{dd}, J$ = 2.0, $8.8 \mathrm{~Hz}, 1 \mathrm{H}), 6.54(\mathrm{~d}, J=8.8 \mathrm{~Hz}, 1 \mathrm{H}), 5.39(\mathrm{~s}, 1 \mathrm{H}), 4.49(\mathrm{~s}, 1 \mathrm{H}), 4.37(\mathrm{br} \mathrm{s}, 1 \mathrm{H}), 4.02(\mathrm{~m}, 2 \mathrm{H})$, $3.72(\mathrm{~s}, 3 \mathrm{H}), 2.27(\mathrm{~s}, 3 \mathrm{H}), 1.11(\mathrm{t}, J=6.8 \mathrm{~Hz}, 3 \mathrm{H}) ;{ }^{13} \mathrm{C} \mathrm{NMR}\left(75 \mathrm{MHz}, \mathrm{CDCl}_{3}\right) \square=198.6,169.6,153.0$, 138.1, 134.2, 134.1, 129.0, 128.3, 126.5, 116.9, 114.0, 111.6, 74.3, 61.8, 61.4, 55.5, 17.7, 13.9; IR (neat, $\left.4 \mathrm{~cm}^{-1}\right): \mathrm{C}=3282,2933,1678,1657,1598,1505,1449,1275,1221,1157,1047,804,693$; HRMS (EI) $\mathrm{m} / \mathrm{z} 357.1275\left[\mathrm{M}^{+}\right.$; calcd for $\left.\mathrm{C}_{20} \mathrm{H}_{23} \mathrm{NO}_{5}, 357.1576\right]$.

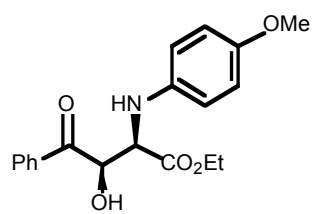

diastereomeric mixture $(d r=2: 1)$; Syn diastereomer: $t_{r}=44.7 \mathrm{~min}$ (major enantiomer) and $65.3 \mathrm{~min}$, (Chiralcel AD, $]=254 \mathrm{~nm}$, heptane: $i-\mathrm{PrOH}=90: 10,1 \mathrm{~mL} / \mathrm{min}$ ); $[\square]_{\mathrm{D}}^{25}+15.3^{\circ}$ (c $0.647, \mathrm{CHCl}_{3}, 84 \%$ ee); 
${ }^{1} \mathrm{H} \mathrm{NMR}\left(300 \mathrm{MHz}, \mathrm{CDCl}_{3}\right) \mathrm{\square}=($ syn isomer) $7.93(\mathrm{~m}, 2 \mathrm{H}), 7.64(\mathrm{~m}, 1 \mathrm{H}), 7.52(\mathrm{~m}, 2 \mathrm{H}), 6.78(\mathrm{~d}, \mathrm{~J}=8.8$ $\mathrm{Hz}, 2 \mathrm{H}), 6.68(\mathrm{~d}, J=8.8 \mathrm{~Hz}, 2 \mathrm{H}), 5.38(\mathrm{~d}, J=2.0 \mathrm{~Hz}, 1 \mathrm{H}), 4.46(\mathrm{~d}, J=2.0 \mathrm{~Hz}, 1 \mathrm{H}), 4.01(\mathrm{~m}, 2 \mathrm{H}), 3.72$ $(\mathrm{s}, 3 \mathrm{H}), 1.11(\mathrm{t}, J=6.8 \mathrm{~Hz}, 3 \mathrm{H}) ;{ }^{13} \mathrm{C}$ NMR $\left(75 \mathrm{MHz}, \mathrm{CDCl}_{3}\right) \square=$ (syn isomer) 198.5, 169.5, 153.4, 140.0, $134.2,134.1,129.1,129.0,128.4,128.3,116.5,114.8,74.2,61.9,61.4,55.6,13.9$; IR (neat, $\left.4 \mathrm{~cm}^{-1}\right): \square=$ $3457,3373,2983,2935,2834,1747,1687,1598,1514,1464,1450,1408,1370,1241,1183,1102$, 1034, 970, 824, 770, 694; HRMS (EI) $\mathrm{m} / \mathrm{z} 343.1423$ [M+; calcd for $\left.\mathrm{C}_{19} \mathrm{H}_{21} \mathrm{NO}_{5}, 343.1420\right]$.

Syn diastereomer: $\mathrm{t}_{\mathrm{r}}=14.4 \mathrm{~min}$ (major enantiomer) and $18.2 \mathrm{~min}$, (Chiralcel $\mathrm{AD}, \mathrm{C}=254 \mathrm{~nm}$, heptane:i-

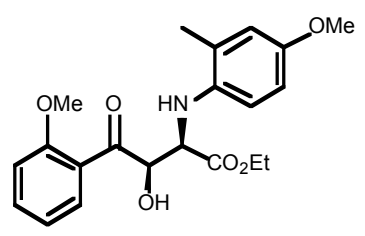

$\mathrm{PrOH}=80: 20,1 \mathrm{~mL} / \mathrm{min}) ;{ }^{1} \mathrm{H}$ NMR $\left(300 \mathrm{MHz}, \mathrm{CDCl}_{3}\right) \mathrm{\square}=$ (syn isomer) 7.85 (dd, $J=1.9,7.8 \mathrm{~Hz}, 1 \mathrm{H}$ ), $7.55(\mathrm{dt}, J=1.9,8.8 \mathrm{~Hz}, 1 \mathrm{H}), 7.08(\mathrm{t}, J=7.8 \mathrm{~Hz}, 1 \mathrm{H}), 7.01(\mathrm{~d}, J=7.8 \mathrm{~Hz}, 1 \mathrm{H}), 6.70(\mathrm{~d}, J=1.9 \mathrm{~Hz}, 1 \mathrm{H})$, $6.61(\mathrm{dd}, J=2.9,8.8 \mathrm{~Hz}, 1 \mathrm{H}), 6.53(\mathrm{~d}, J=8.8 \mathrm{~Hz}, 1 \mathrm{H}), 5.43(\mathrm{~d}, J=3.9 \mathrm{~Hz}, 1 \mathrm{H}), 4.48(\mathrm{~s}, 1 \mathrm{H}), 4.36(\mathrm{br} \mathrm{s}$, $1 \mathrm{H}), 4.10-3.92(\mathrm{~m}, 2 \mathrm{H}), 3.81(\mathrm{~s}, 3 \mathrm{H}), 3.72(\mathrm{~s}, 3 \mathrm{H}), 2.26(\mathrm{~s}, 3 \mathrm{H}), 1.12(\mathrm{t}, J=6.8 \mathrm{~Hz}, 3 \mathrm{H}) ;{ }^{13} \mathrm{C} \mathrm{NMR}$ $\left(75 \mathrm{MHz}, \mathrm{CDCl}_{3}\right) \mathrm{\square}=199.5,170.1,158.4,152.8,138.6,134.9,131.8,126.4,124.4,121.2,116.7,114.2$, 111.6 (2), 78.4, 61.3, 55.6 (2), 17.7, 14.0; IR (neat, $4 \mathrm{~cm}^{-1}$ ): $\mathrm{\square}=3426,2977,2940,2831,1996,1745$, $1667,1598,1512,1486,1465,1438,1291,1245,1224,1184,1163,1097,1052,1021,975,866,803$, 760; HRMS (EI) $m / z 387.1680\left[\mathrm{M}^{+}\right.$; calcd for $\left.\mathrm{C}_{21} \mathrm{H}_{25} \mathrm{NO}_{6}, 387.1682\right]$.

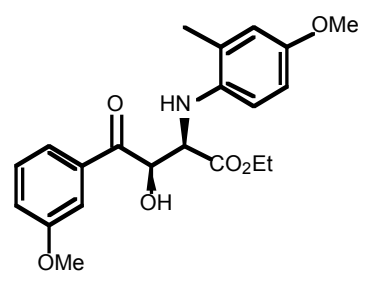

Syn diastereomer: $\mathrm{t}_{\mathrm{r}}=18.6 \mathrm{~min}$ and $27.3 \mathrm{~min}$ (major enantiomer), (Chiralcel OD, $\mathrm{T}=254 \mathrm{~nm}$, heptane: $\mathrm{i}-$ $\mathrm{PrOH}=90: 10,0.9 \mathrm{~mL} / \mathrm{min}) ;{ }^{1} \mathrm{H}$ NMR $\left(300 \mathrm{MHz}, \mathrm{CDCl}_{3}\right) \square=$ (syn isomer) $7.45(\mathrm{~m}, 3 \mathrm{H}), 7.17$ (dd, $J=2.9$, $7.8 \mathrm{~Hz}, 1 \mathrm{H}), 6.71(\mathrm{~d}, J=2.9 \mathrm{~Hz}, 1 \mathrm{H}), 6.63(\mathrm{dd}, J=2.9,8.8 \mathrm{~Hz}, 1 \mathrm{H}), 6.56(\mathrm{~d}, J=7.8 \mathrm{~Hz}, 1 \mathrm{H}), 6.56(\mathrm{~d}, J$ $=7.8 \mathrm{~Hz}, 1 \mathrm{H}), 5.36(\mathrm{~d}, J=1.9 \mathrm{~Hz}, 1 \mathrm{H}), 4.51(\mathrm{~d}, J=2.9 \mathrm{~Hz}, 1 \mathrm{H}), 4.02(\mathrm{~m}, 2 \mathrm{H}), 3.85(\mathrm{~s}, 3 \mathrm{H}), 3.72(\mathrm{~s}, 3$ $\mathrm{H}), 2.27(\mathrm{~s}, 3 \mathrm{H}), 1.13(\mathrm{t}, \mathrm{J}=6.8 \mathrm{~Hz}, 3 \mathrm{H}) ;{ }^{13} \mathrm{C}$ NMR $\left(75 \mathrm{MHz}, \mathrm{CDCl}_{3}\right) \square=$ (syn isomer) 198.5, 169.6, 160.0, 153.0, 138.1, 135.5, 130.0, 126.5, 120.6, 120.4, 116.9, 114.1, 112.7, 111.6, 74.3, 61.9, 61.4, 55.5, $55.4,17.7,13.9 ;{ }^{1} \mathrm{H}$ NMR $\left(300 \mathrm{MHz}, \mathrm{CDCl}_{3}\right) \square=$ minor diastereomer (non-overlapping peaks): 6.37 (dd, $J$ = 2.9, $8.8 \mathrm{~Hz}, 1 \mathrm{H}), 5.95(\mathrm{~d}, J=8.8 \mathrm{~Hz}, 1 \mathrm{H}), 5.54(\mathrm{~s}, 1 \mathrm{H}), 4.24(\mathrm{~m}, 2 \mathrm{H}), 3.81(\mathrm{~s}, 3 \mathrm{H}), 3.64(\mathrm{~s}, 3 \mathrm{H}), 2.12$ $(\mathrm{s}, 3 \mathrm{H}), 1.29(\mathrm{t}, J=6.8 \mathrm{~Hz}, 3 \mathrm{H}) ;{ }^{13} \mathrm{C} \operatorname{NMR}\left(75 \mathrm{MHz}, \mathrm{CDCl}_{3}\right) \square=171.1,120.8,120.7,120.0,116.6,113.7$, 111.3, 17.5, 14.1; IR (neat, $4 \mathrm{~cm}^{-1}$ ): $\mathrm{C}=3426,2938,2833,1996,1746,1687,1593,1582,1511,1483$, 1448, 1432, 1273, 1223, 1192, 1157, 1098, 1047, 867, 790; HRMS (EI) m/z 387.1679 [M+; calcd for $\left.\mathrm{C}_{21} \mathrm{H}_{25} \mathrm{NO}_{6}, 387.1682\right]$. 


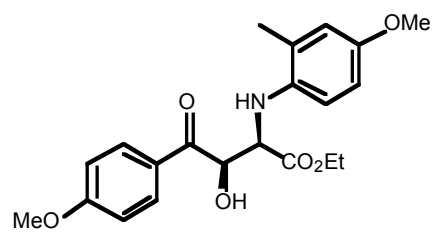

Diastereomeric mixture $(d r=1: 1)$; syn diastereomer: $t_{r}=22.1 \mathrm{~min}$ (major enantiomer) and $29.6 \mathrm{~min}$, (Chiralcel OD, $\square=254 \mathrm{~nm}$, heptane:i-PrOH = 90:10, $0.9 \mathrm{~mL} / \mathrm{min}) ;{ }^{1} \mathrm{H} \mathrm{NMR}\left(300 \mathrm{MHz}, \mathrm{CDCl}_{3}\right) \square=7.94(\mathrm{t}$, $J=8.8 \mathrm{~Hz}, 4 \mathrm{H}), 6.98(\mathrm{dd}, J=3.9,8.8 \mathrm{~Hz}, 4 \mathrm{H}), 6.71(\mathrm{~d}, J=1.9 \mathrm{~Hz}, 1 \mathrm{H}), 6.63(\mathrm{dd}, J=1.9,8.8 \mathrm{~Hz}, 1 \mathrm{H}$ ), $6.58(\mathrm{~d}, J=2.9 \mathrm{~Hz}, 1 \mathrm{H}), 6.54(\mathrm{~d}, J=8.8 \mathrm{~Hz}, 1 \mathrm{H}), 6.40(\mathrm{dd}, J=1.9,8.8 \mathrm{~Hz}, 1 \mathrm{H}), 5.98(\mathrm{~d}, J=8.8 \mathrm{~Hz}, 1$ H), $5.51(\mathrm{~s}, 1 \mathrm{H}), 5.35(\mathrm{~d}, J=5.9 \mathrm{~Hz}, 1 \mathrm{H}), 4.47(\mathrm{~s}, 2 \mathrm{H}), 4.32-4.20(\mathrm{~m}, 3 \mathrm{H}), 4.11-3.94(\mathrm{~m}, 2 \mathrm{H}), 3.88$ (s, $3 \mathrm{H}), 3.87(\mathrm{~s}, 3 \mathrm{H}), 3.72(\mathrm{~s}, 3 \mathrm{H}), 3.64(\mathrm{~s}, 3 \mathrm{H}), 2.27(\mathrm{~s}, 3 \mathrm{H}), 2.12(\mathrm{~s}, 3 \mathrm{H}), 1.29(\mathrm{t}, J=6.8 \mathrm{~Hz}, 3 \mathrm{H})$, $1.10(\mathrm{t}, J=6.8 \mathrm{~Hz}, 3 \mathrm{H}) ;{ }^{13} \mathrm{C} \mathrm{NMR}\left(75 \mathrm{MHz}, \mathrm{CDCl}_{3}\right) \mathrm{Z}=196.6,196.2,171.3,169.7,164.3$ (2), 152.9, 152.6, 138.3, 138.2, 130.9, 130.7, 129.9, 126.9, 126.5, 126.0, 125.8, 116.9, 116.6, 114.3, 114.1, 113.9, 113.5, 111.5, 111.3, 76.6, 73.8, 73.7, 61.9, 61.8, 61.5, 61.3, 55.5, 55.4, 17.7, 17.6, 14.2, 13.9; HRMS (EI) $\mathrm{m} / \mathrm{z} 387.1679\left[\mathrm{M}^{+}\right.$; calcd for $\left.\mathrm{C}_{21} \mathrm{H}_{25} \mathrm{NO}_{6}, 387.1682\right]$.

Anti diastereomer: $\mathrm{t}_{\mathrm{r}}=16.6 \mathrm{~min}$ (major enantiomer) and $19.7 \mathrm{~min}$, (Chiralcel OD, $\square=254 \mathrm{~nm}$, heptane: $\mathrm{i}_{-}$ $\mathrm{PrOH}=90: 10,0.9 \mathrm{~mL} / \mathrm{min}) 55 \%$ ee.

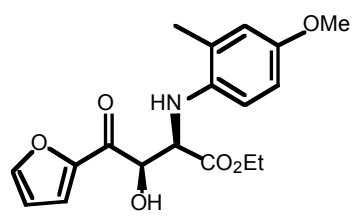

Diastereomeric mixture $(d r=5: 1)$; syn diastereomer: $t_{r}=47.5 \mathrm{~min}$ and $58.7 \mathrm{~min}$ (major enantiomer), (Chiralcel OD, $\square=254 \mathrm{~nm}$, heptane: $\mathrm{P} \mathrm{PrOH}=90: 10,1 \mathrm{~mL} / \mathrm{min}) ;{ }^{1} \mathrm{H} \mathrm{NMR}\left(300 \mathrm{MHz}, \mathrm{CDCl}_{3}\right) \square=($ syn isomer) $7.69(\mathrm{~d}, J=2.0 \mathrm{~Hz}, 1 \mathrm{H}), 7.40(\mathrm{~d}, J=3.9 \mathrm{~Hz}, 1 \mathrm{H}), 6.70(\mathrm{~m}, 3 \mathrm{H}), 6.63(\mathrm{dd}, J=2.0,3.9 \mathrm{~Hz}, 1 \mathrm{H})$, $5.15(\mathrm{~d}, J=3.9 \mathrm{~Hz}, 1 \mathrm{H}), 4.74(\mathrm{~d}, J=3.9 \mathrm{~Hz}, 1 \mathrm{H}), 4.04(\mathrm{~m}, 2 \mathrm{H}), 3.72(\mathrm{~s}, 3 \mathrm{H}), 2.26(\mathrm{~s}, 3 \mathrm{H}), 1.10(\mathrm{t}, J=$ $6.8 \mathrm{~Hz}, 3 \mathrm{H}) ;{ }^{13} \mathrm{C}$ NMR $\left(75 \mathrm{MHz}, \mathrm{CDCl}_{3}\right) \mathrm{\square}=$ (syn isomer) 186.7, 169.9, 152.8, 150.8, 147.1, 138.1, 126.1, $118.9,117.0,113.4,112.9,111.6,74.4,61.5,61.3,55.5,17.7,13.8$; IR (neat, $4 \mathrm{~cm}^{-1}$ ): $\square=3420,3137$, 2981, 1996, 1745, 1674, 1570, 1513, 1465, 1386, 1289, 1224, 1161, 1098, 1020, 883, 768; HRMS (EI) $\mathrm{m} / \mathrm{z} 347.1369\left[\mathrm{M}^{+}\right.$; calcd for $\left.\mathrm{C}_{18} \mathrm{H}_{21} \mathrm{NO}_{6}, 347.1369\right]$.

\section{General procedure for the imine aldol (Mannich-type) reaction of aldimines:}

\section{Preparation of the catalyst:}

To a room temperature solution of ligand $1 \mathrm{a}(10 \mathrm{mg}, 0.015 \mathrm{mmol})$ in THF $(0.3 \mathrm{~mL})$ was added dropwise a solution of diethylzinc ( $30 \mathrm{~L}, 0.030 \mathrm{mmol}, 1.0 \mathrm{M}$ hexanes) and continued to stir for 30 minutes.

\section{Mannich-type (Imine Aldol) reaction:}


To a $-78{ }^{\circ} \mathrm{C}$ suspension of imine $6 \mathrm{~b}(64 \mathrm{mg}, 0.30 \mathrm{mmol})$, 2-hydroxyacetophenone ( $82 \mathrm{mg}, 0.60$ $\mathrm{mmol})$ and crushed $4 \AA$ molecular sieves $(60 \mathrm{mg})$ in THF $(0.6 \mathrm{~mL})$ was added the zinc catalyst from above. After stirring at $-5{ }^{\circ} \mathrm{C}$ for $18-24$ hours, the reaction mixture was diluted with $\mathrm{Et}_{2} \mathrm{O}(6 \mathrm{~mL})$ and then a phosphate buffer $(\mathrm{pH}=7)$ solution $(3 \mathrm{~mL})$. After stirring for 30 minutes, the phases were separated and the aqueous phase was extracted with $\mathrm{Et}_{2} \mathrm{O}(1 \mathrm{~mL} \times 2)$. The combined organics were

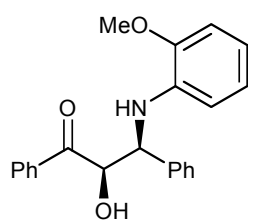

washed with brine $(1.5 \mathrm{~mL})$, dried over $\mathrm{MgSO}_{4}$, filtered and concentrated to give an oil. The crude residue was purified by flash chromatography on silica gel [PE / EtOAc $10 \%$ ] to give $69 \mathrm{mg}(66 \%)$ of imine aldol 7 as a yellow oil.

Syn diastereomer: $\mathrm{t}_{\mathrm{r}}=21.1 \mathrm{~min}$ and $40.0 \mathrm{~min}$ (major), (Chiralcel OD, $\mathrm{Q}=254 \mathrm{~nm}$, heptane: $\mathrm{i}-\mathrm{PrOH}=$ 90:10, $0.9 \mathrm{~mL} / \mathrm{min}$ ); $[\square]_{\mathrm{D}}^{25}-44.8^{\circ}$ (c $0.862, \mathrm{CHCl}_{3},>99 \%$ ee); ${ }^{1} \mathrm{H} \mathrm{NMR}\left(300 \mathrm{MHz}, \mathrm{CDCl}_{3}\right) \square=7.91$ (d, $\mathrm{J}=$ $7.8 \mathrm{~Hz}, 2 \mathrm{H}), 7.67(\mathrm{t}, J=7.8 \mathrm{~Hz}, 1 \mathrm{H}), 7.54(\mathrm{t}, J=7.8 \mathrm{~Hz}, 2 \mathrm{H}), 7.16(\mathrm{~m}, 3 \mathrm{H}), 6.96(\mathrm{~m}, 2 \mathrm{H}), 6.77(\mathrm{dd}, J=$ 1.9, $6.8 \mathrm{~Hz}, 1 \mathrm{H}), 6.63(\mathrm{~m}, 2 \mathrm{H}), 6.31(\mathrm{~m}, 1 \mathrm{H}), 5.64(\mathrm{~s}, 1 \mathrm{H}), 4.95(\mathrm{~s}, 1 \mathrm{H}), 3.91(\mathrm{~s}, 3 \mathrm{H}), 3.70(\mathrm{br} \mathrm{s}, 1 \mathrm{H})$; ${ }^{13} \mathrm{C} \mathrm{NMR}\left(75 \mathrm{MHz}, \mathrm{CDCl}_{3}\right) \square=198.7,147.1,136.4,136.0,134.2,129.1,128.6,128.0,127.8,127.4$, $120.9,117.1,111.2,109.5,76.3,60.2,55.4$;

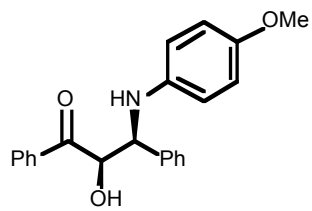

Diastereomeric mixture $(\mathrm{dr}=5: 1) ;[\square]^{25}-9.58^{\circ}$ (c $0.748, \mathrm{CHCl}_{3}, 99 \%$ ee); syn diastereomer: $\mathrm{t}_{\mathrm{r}}=18.1 \mathrm{~min}$ (major enantiomer) and $20.9 \mathrm{~min}$, (Chiralcel OD, $\mathrm{C}=254 \mathrm{~nm}$, heptane: $i-\mathrm{PrOH}=90: 10,0.9 \mathrm{~mL} / \mathrm{min}$ ); ${ }^{1} \mathrm{H}$ $\operatorname{NMR}\left(300 \mathrm{MHz}, \mathrm{CDCl}_{3}\right) \square=7.88(\mathrm{~m}, 3 \mathrm{H}), 7.52(\mathrm{t}, J=7.8 \mathrm{~Hz}, 3 \mathrm{H}), 7.14(\mathrm{~m}, 2 \mathrm{H}), 6.90(\mathrm{~m}, 2 \mathrm{H}), 6.66(\mathrm{~d}, J$ $=8.8 \mathrm{~Hz}, 2 \mathrm{H}), 6.54(\mathrm{~d}, J=8.8 \mathrm{~Hz}, 2 \mathrm{H}), 5.58(\mathrm{br} \mathrm{s}, 1 \mathrm{H}), 4.82(\mathrm{dd}, J=1.9,6.8 \mathrm{~Hz}, 1 \mathrm{H}), 3.66(\mathrm{~s}, 3 \mathrm{H})$; minor diastereomer (non-overlapping peaks): $7.64(\mathrm{~m}, 4 \mathrm{H}), 7.37(\mathrm{~d}, J=7.8 \mathrm{~Hz}, 2 \mathrm{H}), 7.31(\mathrm{t}, J=7.8 \mathrm{~Hz}, 2$ $\mathrm{H}), 6.31(\mathrm{~d}, J=8.8 \mathrm{~Hz}, 2 \mathrm{H}), 5.32(\mathrm{~d}, J=2.9 \mathrm{~Hz}, 1 \mathrm{H}), 3.61(\mathrm{~s}, 3 \mathrm{H}) ;{ }^{13} \mathrm{C}$ NMR $\left(75 \mathrm{MHz}, \mathrm{CDCl}_{3}\right) \square=($ both diastereomers) 200.0, 198.7, 152.3, 152.2, 140.2, 140.1, 136.4, 134.2, 134.1, 133.9, 133.8, 129.1, 128.9, 128.6, 128.5, 128.4, 128.0, 127.8, 127.6, 127.5, 127.4, 126.9, 115.4, 115.3, 114.7, 114.5, 76.0, 61.3, 60.7, 55.5; IR (neat, $4 \mathrm{~cm}^{-1}$ ): $\mathrm{T}=3390,3062,3029,2932,2833,1683,1619,1598,1580,1513,1451$, 1407, 1241, 1180, 1103, 1075, 1036, 1002, 988, 821, 790, 759, 699, 668; HRMS (EI) m/z 347.1529 [M+; calcd for $\mathrm{C}_{22} \mathrm{H}_{21} \mathrm{NO}_{3}, 347.1521$ ].

Anti diastereomer: $\mathrm{t}_{\mathrm{r}}=27.2 \mathrm{~min}$ and $34.9 \mathrm{~min}$ (major enantiomer), (Chiralcel OD, $\square=254 \mathrm{~nm}$, heptane: ${ }_{-}$ $\mathrm{PrOH}=90: 10,0.9 \mathrm{~mL} / \mathrm{min}), 95 \%$ ee. 


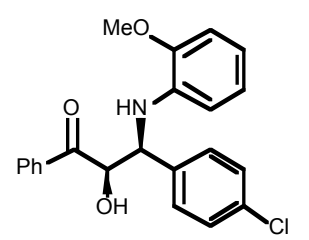

Syn diastereomer: $\mathrm{t}_{\mathrm{r}}=27.1 \mathrm{~min}$ and $47.9 \mathrm{~min}$ (major enantiomer), (Chiralcel OD, $\mathrm{V}=254 \mathrm{~nm}$, heptane:i-PrOH = 90:10, $0.9 \mathrm{~mL} / \mathrm{min}$ ); $[\square]^{25} \mathrm{D}-104.5^{\circ}$ (c $0.633, \mathrm{CHCl}_{3},>99 \%$ ee); ${ }^{1} \mathrm{H} \mathrm{NMR}(300 \mathrm{MHz}$, $\left.\mathrm{CDCl}_{3}\right) \square=7.90(\mathrm{dd}, J=1.9,8.8 \mathrm{~Hz}, 2 \mathrm{H}), 7.68(\mathrm{t}, J=7.8 \mathrm{~Hz}, 1 \mathrm{H}), 7.54(\mathrm{t}, J=7.8 \mathrm{~Hz}, 2 \mathrm{H}), 7.10(\mathrm{~d}, J=$ $8.8 \mathrm{~Hz}, 2 \mathrm{H}), 6.85(\mathrm{~d}, J=8.8 \mathrm{~Hz}, 2 \mathrm{H}), 6.76(\mathrm{~m}, 1 \mathrm{H}), 6.62(\mathrm{~m}, 2 \mathrm{H}), 6.23(\mathrm{~m}, 1 \mathrm{H}), 5.60(\mathrm{~d}, J=3.9 \mathrm{~Hz}, 1$ $\mathrm{H}), 5.50(\mathrm{br} \mathrm{s}, 1 \mathrm{H}), 4.88(\mathrm{~d}, J=2.9 \mathrm{~Hz}, 1 \mathrm{H}), 3.90(\mathrm{~s}, 3 \mathrm{H}), 3.68(\mathrm{~d}, J=7.8 \mathrm{~Hz}, 1 \mathrm{H}) ;{ }^{13} \mathrm{C} \mathrm{NMR}(75 \mathrm{MHz}$, $\left.\mathrm{CDCl}_{3}\right) \square=198.5,147.2,135.7,135.1,134.5,134.0,133.6,129.3,128.8,128.6,128.3,120.8,117.4$, 111.3, 109.6, 76.1, 59.8, 55.5; IR (neat, $4 \mathrm{~cm}^{-1}$ ): $\mathrm{V}=3429$, 3064, 2936, 2835, 1684, 1600, 1579, 1512, 1490, 1456, 1430, 1409, 1343, 1270, 1244, 1225, 1178, 1134, 1108, 1093, 1050, 1028, 1014, 990, 952, $880,825,789,740,695,670$.

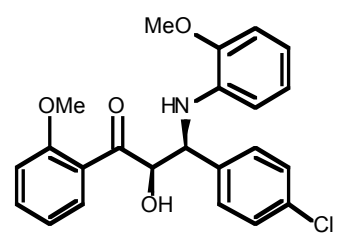

Syn diastereomer: $\mathrm{t}_{\mathrm{r}}=14.4 \mathrm{~min}$ (major enantiomer) and $17.8 \mathrm{~min}$, (Chiralcel OD, $\square=254 \mathrm{~nm}$, heptane: $j-$ $\mathrm{PrOH}=90: 10,0.9 \mathrm{~mL} / \mathrm{min}$ ); $[\square]^{25}{ }_{\mathrm{D}}+170.6^{\circ}$ (c $0.942, \mathrm{CHCl}_{3}, 98.6 \%$ ee); ${ }^{1} \mathrm{H} \mathrm{NMR}\left(300 \mathrm{MHz}, \mathrm{CDCl}_{3}\right) \square=$ $7.66(\mathrm{~d}, J=7.8 \mathrm{~Hz}, 1 \mathrm{H}), 7.59(\mathrm{dt}, J=1.9,8.8 \mathrm{~Hz}, 1 \mathrm{H}), 7.09(\mathrm{~d}, J=7.8 \mathrm{~Hz}, 2 \mathrm{H}), 7.04(\mathrm{~m}, 2 \mathrm{H}), 6.90(\mathrm{~d}, J$ $=7.8 \mathrm{~Hz}, 2 \mathrm{H}), 6.75(\mathrm{dd}, J=1.9,7.8 \mathrm{~Hz}, 1 \mathrm{H}), 6.61(\mathrm{dt}, J=1.9,6.8 \mathrm{~Hz}, 2 \mathrm{H}), 6.27(\mathrm{dd}, J=1.9,7.8 \mathrm{~Hz}, 1$ $\mathrm{H}), 5.71(\mathrm{dd}, J=2.9,6.8 \mathrm{~Hz}, 1 \mathrm{H}), 5.54(\mathrm{br} \mathrm{s}, 1 \mathrm{H}), 4.84(\mathrm{br} \mathrm{s}, 1 \mathrm{H}), 3.89(\mathrm{~s}, 3 \mathrm{H}), 3.87(\mathrm{~s}, 3 \mathrm{H}) ;{ }^{13} \mathrm{C} \mathrm{NMR}$ $\left(75 \mathrm{MHz}, \mathrm{CDCl}_{3}\right) \mathrm{\square}=199.1,159.0,147.1,136.2,135.9,135.4,133.2,131.8,129.2,128.5,128.2,127.9$, 124.2, 121.3, 120.8, 116.9, 111.9, 111.1, 109.5, 79.7, 58.7, 55.7, 55.4; IR (neat, $4 \mathrm{~cm}^{-1}$ ): $\square=3432,3068$, $3006,2942,2839,1666,1598,1513,1486,1456,1435,1408,1344,1293,1245,1223,1178,1164$, $1120,1092,1052,1023,978,881,825,805,781,760,739,653$;

Anti diastereomer: $\mathrm{t}_{\mathrm{r}}=13.0 \mathrm{~min}$ (major enantiomer) and $28.7 \mathrm{~min}$, (Chiralcel OD, $\square=254 \mathrm{~nm}$, heptane: $\mathrm{i}_{-}$ $\mathrm{PrOH}=90: 10,0.9 \mathrm{~mL} / \mathrm{min})$.

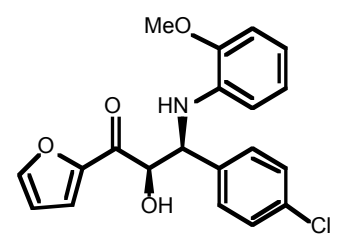

Syn diastereomer: $\mathrm{t}_{\mathrm{r}}=31.0 \mathrm{~min}$ and $49.1 \mathrm{~min}$ (major enantiomer), (Chiralcel OD, $\square=254 \mathrm{~nm}$, heptane: $\mathrm{i}-$ $\mathrm{PrOH}=90: 10,0.9 \mathrm{~mL} / \mathrm{min}$ ); $[\square]^{25}-125.19^{\circ}$ (c 1.0, $\mathrm{CHCl}_{3}, 97.4 \%$ ee); ${ }^{1} \mathrm{H} \mathrm{NMR}\left(300 \mathrm{MHz}, \mathrm{CDCl}_{3}\right) \square=7.68$ 
(s, $1 \mathrm{H}), 7.30$ (d, J = 3.9 Hz, $1 \mathrm{H}), 7.14$ (d, J = 7.8 Hz, $2 \mathrm{H}), 6.99$ (d, J = 7.8 Hz, 2 H), 6.77 (dd, J = 1.9, 6.8 $\mathrm{Hz}, 1 \mathrm{H}), 6.70-6.60(\mathrm{~m}, 3 \mathrm{H}), 6.37(\mathrm{dd}, J=1.9,6.8 \mathrm{~Hz}, 1 \mathrm{H}), 5.57(\mathrm{br} \mathrm{s}, 1 \mathrm{H}), 5.35(\mathrm{~s}, 1 \mathrm{H}), 5.05(\mathrm{~d}, J=$ $2.9 \mathrm{~Hz}, 1 \mathrm{H}), 3.89(\mathrm{~s}, 3 \mathrm{H}), 3.59(\mathrm{br} \mathrm{d}, J=5.9 \mathrm{~Hz}, 1 \mathrm{H}) ;{ }^{13} \mathrm{C} \mathrm{NMR}\left(75 \mathrm{MHz}, \mathrm{CDCl}_{3}\right) \square=186.7,150.5$, 147.5, 147.1, 135.7, 135.6, 133.5, 128.7, 128.4, 120.8, 119.4, 117.3, 113.0, 111.1, 109.5, 76.1, 59.5, 55.4; IR (neat, $4 \mathrm{~cm}^{-1}$ ): $\mathrm{Z}=3422,3133,2936,2836,1671,1602,1570,1512,1490,1458,1430,1385$, 1344, 1286, 1247, 1225, 1176, 1134, 1110, 1090, 1050, 1023, 909, 883, 859, 794, 769, 739;

\section{Preparation of oxazolidinone (8):}

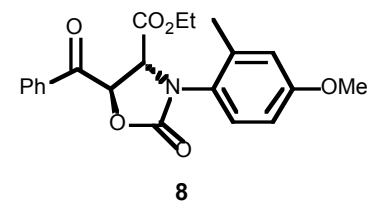

To a $-78{ }^{\circ} \mathrm{C}$ solution of amino alcohol $5(220 \mathrm{mg}, 0.615 \mathrm{mmol})$ in $\mathrm{CH}_{2} \mathrm{Cl}_{2}(6 \mathrm{~mL})$ were added $i-$ $\operatorname{Pr}_{2} \mathrm{NEt}\left(0.32 \mathrm{~mL}, 1.85 \mathrm{mmol}\right.$ ) and a solution of triphosgene $(270 \mathrm{mg}, 0.923 \mathrm{mmol})$ in $\mathrm{CH}_{2} \mathrm{Cl}_{2}(1 \mathrm{~mL})$ dropwise. The reaction was slowly allowed to warm to room temperature (overnight) and then quenched with $\mathrm{NH}_{4} \mathrm{OH}(30 \%, 1 \mathrm{~mL})$ and water $(1 \mathrm{~mL})$. $\mathrm{Et}_{2} \mathrm{O}(15 \mathrm{~mL})$ was added, and the phases were separated. The organic phase was washed with brine $(1 \mathrm{~mL})$, dried over $\mathrm{MgSO}_{4}$, filtered and concentrated to give $0.46 \mathrm{~g}$ of a yellow oil. The residue was purified by flash chromatography on silica gel [PE / EtOAc $15 \%$ ○ $25 \%$ ] to give $230 \mathrm{mg}$ (60\%) of oxazolidinone 8 as a yellow oil. $[\square]^{25}{ }_{\mathrm{D}}-33.9^{\circ}$ (c $\left.0.923, \mathrm{CHCl}_{3}\right) ;{ }^{1} \mathrm{H}$ NMR $\left(500 \mathrm{MHz}, \mathrm{CDCl}_{3}\right) \square=8.11(\mathrm{dd}, J=1.0,8.3 \mathrm{~Hz}, 2 \mathrm{H}), 7.66(\mathrm{t}, J=7.3 \mathrm{~Hz}, 1 \mathrm{H}), 7.53(\mathrm{t}, J=7.8 \mathrm{~Hz}, 2 \mathrm{H})$, $7.29(\mathrm{~d}, J=8.3 \mathrm{~Hz}, 1 \mathrm{H}), 6.74(\mathrm{~m}, 2 \mathrm{H}), 5.77(\mathrm{~d}, J=3.9 \mathrm{~Hz}, 1 \mathrm{H}), 5.20(\mathrm{~d}, J=3.4 \mathrm{~Hz}, 1 \mathrm{H}), 4.21$ (q, $J=6.8$ $\mathrm{Hz}, 2 \mathrm{H}), 3.77(\mathrm{~s}, 3 \mathrm{H}), 2.25(\mathrm{~s}, 3 \mathrm{H}), 1.21(\mathrm{t}, \mathrm{J}=6.8 \mathrm{~Hz}, 3 \mathrm{H}) ;{ }^{13} \mathrm{C} \mathrm{NMR}\left(75 \mathrm{MHz}, \mathrm{CDCl}_{3}\right) \square=191.0,169.2$, 159.5, 154.6, 138.0, 134.7, 133.1, 129.7, 129.4, 129.0, 126.8, 116.2, 112.1, 75.7, 62.5, 60.2, 55.3, 18.0, 13.9; IR (neat, $4 \mathrm{~cm}^{-1}$ ): $\mathrm{\square}=3065,2978,2936,2850,1775,1694,1608,1598,1581,1506,1450,1421$, 1395, 1285, 1230, 1163, 1142, 1103, 1021, 984, 960, 850, 817, 754, 729, 690, 657, 633; ; HRMS (EI) m/z $383.1357\left[\mathrm{M}^{+}\right.$; calcd for $\left.\mathrm{C}_{21} \mathrm{H}_{21} \mathrm{NO}_{6}, 383.1369\right]$.

CAN promoted nitrogen dearylation to give oxazolidinone (9):

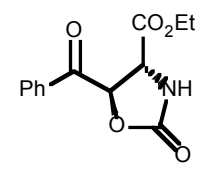

9

To a cold $\left(0{ }^{\circ} \mathrm{C}\right)$ solution of aryl carbamate $8(420 \mathrm{mg}, 1.05 \mathrm{mmol})$ in $\mathrm{CH}_{3} \mathrm{CN}(10 \mathrm{~mL})$ was added dropwise a solution of ceric ammonium nitrate (CAN) $(2.3 \mathrm{~g}, 4.20 \mathrm{mmol})$ in water $(13 \mathrm{~mL})$ over 5 minutes. The ice bath was removed and stirred at room temperature for 60 minutes. The reaction was quenched with saturated aqueous solutions of $\mathrm{Na}_{2} \mathrm{~S}_{2} \mathrm{O}_{3}(13 \mathrm{~mL})$ and $\mathrm{NaHCO}_{3}(10 \mathrm{~mL})$. The reaction mixture was extracted with EtOAc $(25 \mathrm{~mL} \times 3)$. The combined organics were washed with brine $(5 \mathrm{~mL} \times 2)$, dried over $\mathrm{MgSO}_{4}$, filtered and concentrated to give a yellow oil. Purification by flash chromatography on silica gel [PE / EtOAc $25 \%$ ] afforded $125 \mathrm{mg}$ (44\%) of the dearylated oxazolidinone 9 as a yellow oil. $[\square]^{25}-39.5^{\circ}$ 
(c 0.88, $\left.\mathrm{CHCl}_{3}\right) ;{ }^{1} \mathrm{H}$ NMR $\left(300 \mathrm{MHz}, \mathrm{CDCl}_{3}\right) \mathrm{\square}=8.07$ (d, $\left.J=8.8 \mathrm{~Hz}, 2 \mathrm{H}\right), 7.64(\mathrm{t}, J=7.8 \mathrm{~Hz}, 1 \mathrm{H}), 7.50$ $(\mathrm{m}, 2 \mathrm{H}), 5.86(\mathrm{~d}, J=3.9 \mathrm{~Hz}, 1 \mathrm{H}), 5.79(\mathrm{br} \mathrm{s}, 1 \mathrm{H}), 4.92(\mathrm{~d}, J=3.9 \mathrm{~Hz}, 1 \mathrm{H}), 4.29(\mathrm{q}, J=6.8 \mathrm{~Hz}, 2 \mathrm{H})$, 1.31 (t, $J=6.8 \mathrm{~Hz}, 3 \mathrm{H}) ;{ }^{13} \mathrm{C}$ NMR $\left(75 \mathrm{MHz}, \mathrm{CDCl}_{3}\right) \mathrm{\square}=190.8,169.5,156.3,134.7,133.2,129.6,128.9$, 77.8, 62.8, 54.2, 14.1; IR (neat, $4 \mathrm{~cm}^{-1}$ ): $\mathrm{C}=3355,2986,2926,2850,1778,1698,1597,1450,1374$, 1228, 1109, 1021, 975, 758, 690; ; HRMS (EI) $\mathrm{m} / \mathrm{z} 263.0786$ [M+; calcd for $\left.\mathrm{C}_{13} \mathrm{H}_{13} \mathrm{NO}_{5}, 263.0794\right]$.

Baeyer-Villiger oxidation of (9) to give phenyl ester (10):

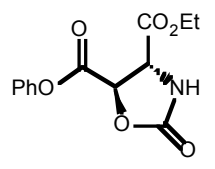

10

To a flame dried flask with crushed molecular sieves $4 \AA(300 \mathrm{mg})$ were added trans-N,N-bis $(p$ toluenesulfonyl)-1,2-cyclohexanediamine (128 mg, $0.304 \mathrm{mmol}$ ) and $\mathrm{CH}_{2} \mathrm{Cl}_{2}(2 \mathrm{~mL}$ ). The suspension was lowered to $-20{ }^{\circ} \mathrm{C}$ and then a solution of $\mathrm{SnCl}_{4}\left(0.30 \mathrm{~mL}, 1.0 \mathrm{M} \mathrm{CH}_{2} \mathrm{Cl}_{2}, 0.30 \mathrm{mmol}\right)$ and bis(trimethylsilyl) peroxide (BTSP) $(0.26 \mathrm{~mL}, 1.22 \mathrm{mmol})$ were added. After stirring for 5 minutes, a solution of aryl ketone 9 (80 mg, $0.304 \mathrm{mmol})$ in $\mathrm{CH}_{2} \mathrm{Cl}_{2}(3 \mathrm{~mL})$ was added. After stirring for an additional 2 hours between -20 ${ }^{\circ} \mathrm{C}$ and $-10{ }^{\circ} \mathrm{C}$, the reaction was quenched with a saturated aqueous solution of $\mathrm{Na}_{2} \mathrm{~S}_{2} \mathrm{O}_{3}(2 \mathrm{~mL})$ and $\mathrm{NaHCO}_{3}(2 \mathrm{~mL})$. EtOAc $(15 \mathrm{~mL})$ was added and the phases were separated. The organics were washed with water $(2 \mathrm{~mL})$ and brine $(2 \mathrm{~mL})$, dried over $\mathrm{Na}_{2} \mathrm{SO}_{4}$, filtered and concentrated to give a yellow solid. EtOAc was added to the solid residue and decanted. Concentration of the solvent and purification by flash chromatography on silica gel [PE / EtOAc 25\%] afforded $55 \mathrm{mg} \mathrm{(65 \% )} \mathrm{of} \mathrm{the} \mathrm{desired} \mathrm{Bayer-Villager}$ adduct 10 as an oil. $[\square]^{25}-18.1^{\circ}$ (c $\left.0.40, \mathrm{CHCl}_{3}\right) ;{ }^{1} \mathrm{H}$ NMR $\left(300 \mathrm{MHz}, \mathrm{CDCl}_{3}\right) \square=7.40$ (t, $J=7.8 \mathrm{~Hz}, 2 \mathrm{H}$ ), $7.29(\mathrm{dd}, J=3.9,7.8 \mathrm{~Hz}, 1 \mathrm{H}), 7.14(\mathrm{~d}, J=7.8 \mathrm{~Hz}, 2 \mathrm{H}), 5.81(\mathrm{~s}, 1 \mathrm{H}), 5.38(\mathrm{~d}, J=3.9 \mathrm{~Hz}, 1 \mathrm{H}), 4.66(\mathrm{~d}, J$ $=3.9 \mathrm{~Hz}, 1 \mathrm{H}$ ), $4.33(\mathrm{q}, J=6.8 \mathrm{~Hz}, 2 \mathrm{H}), 1.34(\mathrm{t}, J=6.8 \mathrm{~Hz}, 3 \mathrm{H}) ;{ }^{13} \mathrm{C} \mathrm{NMR}\left(75 \mathrm{MHz}, \mathrm{CDCl}_{3}\right) \square=168.5$, 166.1, 149.9, 129.6, 127.2, 126.6, 120.9, 74.8, 63.0, 56.5, 14.0; IR (neat, $4 \mathrm{~cm}^{-1}$ ): $\square=3334,2925,2854$, $1784,1493,1375,1214,1189,1094,1023,932,756,689$.

\section{Determination of absolute stereochemistry:}

Preparation of TBS-silyl ether (11):

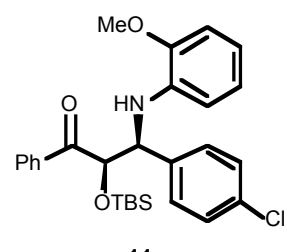

To a cold $\left(0^{\circ} \mathrm{C}\right)$ solution of amino alcohol $7(0.45 \mathrm{~g}, 1.20 \mathrm{mmol})$ in $\mathrm{CH}_{2} \mathrm{Cl}_{2}(5 \mathrm{~mL})$ were added 2,6-lutidine $(0.29 \mathrm{~mL}, 2.5 \mathrm{mmol})$ and TBSOTf $(0.54 \mathrm{~mL}, 2.4 \mathrm{mmol})$. After stirring for 60 minutes at $0{ }^{\circ} \mathrm{C}$, the reaction was quenched with water $(1 \mathrm{~mL})$ and a saturated aqueous $\mathrm{NH}_{4} \mathrm{Cl}$ solution $(1 \mathrm{~mL}) . \mathrm{Et}_{2} \mathrm{O}(10$ $\mathrm{mL}$ ) was added and the phases separated. The organic phase was washed with brine $(1 \mathrm{~mL})$, dried over $\mathrm{MgSO}_{4}$, filtered and concentrated to give $0.9 \mathrm{~g}$ of a yellow oil. Purification by flash chromatography on 
silica gel [PE / EtOAc 5\%] afforded $0.46 \mathrm{~g}(79 \%)$ of TBS-protected alcohol 11 as an oil. $[\square]^{25}-55.40^{\circ}$ (c 0.740, $\left.\mathrm{CHCl}_{3}\right) ;{ }^{1} \mathrm{H}$ NMR $\left(300 \mathrm{MHz}, \mathrm{CDCl}_{3}\right) \square=7.82(\mathrm{~d}, J=7.8 \mathrm{~Hz}, 2 \mathrm{H}), 7.52(\mathrm{t}, J=7.8 \mathrm{~Hz}, 1 \mathrm{H}), 7.38(\mathrm{t}, J$ $=7.8 \mathrm{~Hz}, 2 \mathrm{H}), 7.17(\mathrm{~s}, 4 \mathrm{H}), 6.71(\mathrm{dd}, J=1.9,7.8 \mathrm{~Hz}, 1 \mathrm{H}), 6.60(\mathrm{~m}, 2 \mathrm{H}), 6.27(\mathrm{dd}, J=1.9,7.8 \mathrm{~Hz}, 1 \mathrm{H})$, $5.30(\mathrm{~d}, J=8.8 \mathrm{~Hz}, 1 \mathrm{H}), 5.09$ (d, J = 4.9 Hz, $1 \mathrm{H}$ ), 4.86 (dd, J = 5.9, $8.8 \mathrm{~Hz}, 1 \mathrm{H}), 3.84(\mathrm{~s}, 3 \mathrm{H}), 0.88$ (s, 9 $\mathrm{H}),-0.10$ (s, $3 \mathrm{H}),-0.17$ (s, $3 \mathrm{H}) ;{ }^{13} \mathrm{C}$ NMR $\left(75 \mathrm{MHz}, \mathrm{CDCl}_{3}\right) \mathrm{Z}=199.9,147.1,137.3,135.5,133.4,133.1$, $130.9,129.4,129.3,129.0,128.4,128.2,120.9,117.1,111.3,109.5,80.8,60.0,55.4,25.7,18.2,-4.9$, -5.8; IR (neat, $4 \mathrm{~cm}^{-1}$ ): $\mathrm{\square}=3412,3066,2955,2930,2857,1704,1673,1602,1513,1491,1456,1430$, $1254,1224,1179,1133,1092,1029,980,878,836,781,736,692$.

\section{Nitrogen dearylation to give primary amine (12):}

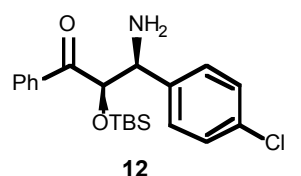

To a cold $\left(0^{\circ} \mathrm{C}\right)$ solution of amine $11(360 \mathrm{mg}, 0.726 \mathrm{mmol})$ in $\mathrm{CH}_{3} \mathrm{CN}(7 \mathrm{~mL})$ was added dropwise a solution of ceric ammonium nitrate (CAN) $(1.6 \mathrm{~g}, 2.90 \mathrm{mmol})$ in water $(9 \mathrm{~mL})$ over 5 minutes. After stirring for 10 minutes at $0{ }^{\circ} \mathrm{C}$, the reaction was quenched with a saturated aqueous $\mathrm{Na}_{2} \mathrm{~S}_{2} \mathrm{O}_{3}$ solution ( $8 \mathrm{~mL}$ ). The reaction mixture was extracted with EtOAc $(20 \mathrm{~mL} \times 4)$. The combined organics were washed with a saturated aqueous $\mathrm{NaHCO}_{3}$ solution $(5 \mathrm{~mL} \times 2)$ and brine $(5 \mathrm{~mL} \times 2)$, dried over $\mathrm{MgSO}_{4}$, filtered and concentrated to give a yellow oil. Purification by flash chromatography on silica gel [PE / EtOAc $25 \%$ ] afforded $200 \mathrm{mg}(71 \%)$ of primary amine 12 as an oil. [प] $]_{\mathrm{D}}^{25}-59.8^{\circ}$ (c $\left.0.50, \mathrm{CHCl}_{3}\right) ;{ }^{1} \mathrm{H} \mathrm{NMR}(300 \mathrm{MHz}$, $\left.\mathrm{CDCl}_{3}\right) \mathrm{D}=7.97(\mathrm{~d}, J=7.8 \mathrm{~Hz}, 2 \mathrm{H}), 7.53(\mathrm{t}, J=7.8 \mathrm{~Hz}, 1 \mathrm{H}), 7.40(\mathrm{t}, J=7.8 \mathrm{~Hz}, 2 \mathrm{H}), 7.23(\mathrm{~s}, 4 \mathrm{H}), 4.74$ (d, $J=6.8 \mathrm{~Hz}, 1 \mathrm{H}$ ), 4.31 (d, J = 6.8 Hz, $1 \mathrm{H}$ ), 1.80 (br s, $2 \mathrm{H}), 0.75$ (s, $9 \mathrm{H}$ ), -0.21 (s, $3 \mathrm{H}),-0.24$ (s, $3 \mathrm{H}$ ); ${ }^{13} \mathrm{C} \mathrm{NMR}\left(75 \mathrm{MHz}, \mathrm{CDCl}_{3}\right) \mathrm{\square}=200.1,140.8,135.4,133.3,133.2,129.2,128.9,128.4,128.2,100.3,82.3$, $59.3,25.6,18.0,-5.0,-5.6$; IR (neat, $4 \mathrm{~cm}^{-1}$ ): $\square=3394,3341,2930,2857,1680,1597,1492,1471,1448$, 1411, 1254, 1114, 1094, 1015, 837, 779, 691.

\section{General procedure for the preparation of the O-methyl mandelate amides:}

To a solution of amine 12 (25 mg, $0.064 \mathrm{mmol})$, (S)-O-methyl mandelic acid (11 mg, $0.067 \mathrm{mmol}$ ) and HOBT $(9 \mathrm{mg}, 0.067 \mathrm{mmol})$ in $\mathrm{CH}_{2} \mathrm{Cl}_{2}(0.6 \mathrm{~mL})$ were added i-Pr $\mathrm{PEt}_{2}(0.012 \mathrm{~mL}, 0.070 \mathrm{mmol})$ and $\mathrm{EDCl} \bullet \mathrm{HCl}(13 \mathrm{mg}, 0.067 \mathrm{mmol})$. After stirring for 20 minutes at room tempature, the reaction was diluted with EtOAc $(2 \mathrm{~mL})$ and then washed with $1 \mathrm{M} \mathrm{HCl}(0.2 \mathrm{~mL} \times 2), \mathrm{NaHCO}_{3}$ (aq. sat.) $(0.2 \mathrm{~mL} \times 2)$, water $(0.2 \mathrm{~mL})$ and brine $(0.4 \mathrm{~mL})$. The organics were dried over $\mathrm{MgSO}_{4}$, filtered and concentrated to give an oil. Purification by flash chromatography on silica gel [PE / EtOAc 5\%] afforded $30 \mathrm{mg}(90 \%)$ of the mandelate amide derived from the (S)-acid.

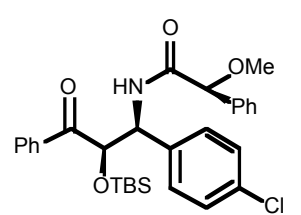

via S-acid 
$[\square]^{25}+41.5^{\circ}$ (c 0.925, $\left.\mathrm{CHCl}_{3}\right) ;{ }^{1} \mathrm{H}$ NMR $\left(300 \mathrm{MHz}, \mathrm{CDCl}_{3}\right) \mathrm{C}=7.88(\mathrm{~d}, J=6.8 \mathrm{~Hz}, 2 \mathrm{H}), 7.65$ (d, J = 8.8 $\mathrm{Hz}, 1 \mathrm{H}), 7.53(\mathrm{t}, J=7.8 \mathrm{~Hz}, 1 \mathrm{H}), 7.41(\mathrm{~d}, J=7.8 \mathrm{~Hz}, 2 \mathrm{H}), 7.29(\mathrm{~s}, 4 \mathrm{H}), 7.15(\mathrm{~d}, J=8.8 \mathrm{~Hz}, 2 \mathrm{H}), 7.03$ $(\mathrm{d}, J=7.8 \mathrm{~Hz}, 2 \mathrm{H}), 5.45(\mathrm{~d}, J=4.9 \mathrm{~Hz}, 1 \mathrm{H}), 5.39$ (dd, $J=3.9,7.8 \mathrm{~Hz}, 1 \mathrm{H}), 4.62(\mathrm{~s}, 1 \mathrm{H}), 3.35(\mathrm{~s}, 3 \mathrm{H})$, 0.95 (s, $9 \mathrm{H}), 0.0$ (s, $6 \mathrm{H}) ;{ }^{13} \mathrm{C}$ NMR $\left(75 \mathrm{MHz}, \mathrm{CDCl}_{3}\right) \mathrm{V}=198.0,169.9,136.5,135.1,135.0,134.0,133.5$, 129.4, 128.8, 128.6, 128.4, 127.1, 124.7, 83.6, 77.8, 57.0, 55.5, 25.7, 18.4, -4.7, -5.7; IR (neat, $4 \mathrm{~cm}^{-1}$ ): $\square$ $=3424,2930,2857,1682,1598,1493,1471,1448,1255,1197,1141,1095,1016,982,837,781,697$.

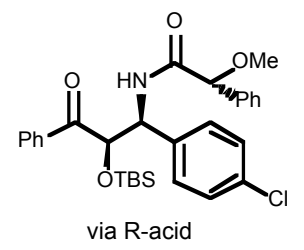

$[\square]^{25}-37.0^{\circ}$ (c 1.0, $\left.\mathrm{CHCl}_{3}\right) ;{ }^{1} \mathrm{H}$ NMR $\left(300 \mathrm{MHz}, \mathrm{CDCl}_{3}\right) \mathrm{\square}=7.79$ (d, $\left.J=7.8 \mathrm{~Hz}, 2 \mathrm{H}\right), 7.71$ (d, $J=7.8 \mathrm{~Hz}, 1$ H), 7.51 (t, $J=7.8 \mathrm{~Hz}, 1 \mathrm{H}), 7.36(\mathrm{~m}, 4 \mathrm{H}), 7.19(\mathrm{~d}, J=8.8 \mathrm{~Hz}, 2 \mathrm{H}), 7.10$ (d, J = 8.8 Hz, $2 \mathrm{H}$ ), 5.33 (dd, $J$ $=4.9,7.8 \mathrm{~Hz}, 1 \mathrm{H}), 5.24(\mathrm{~d}, J=4.9 \mathrm{~Hz}, 1 \mathrm{H}), 4.56(\mathrm{~s}, 1 \mathrm{H}), 3.34(\mathrm{~s}, 3 \mathrm{H}), 0.92(\mathrm{~s}, 9 \mathrm{H}),-0.05(\mathrm{~s}, 3 \mathrm{H}),-0.08$ $(\mathrm{s}, 3 \mathrm{H}) ;{ }^{13} \mathrm{C}$ NMR $\left(75 \mathrm{MHz}, \mathrm{CDCl}_{3}\right) \square=198.1,169.8,136.5,135.9,135.0,133.9,133.5,129.4,128.7$, $128.6,128.5,128.4,127.0,83.5,78.5,57.2,55.4,25.7,18.3,-4.7,-5.6$. 